\section{A: NOD2}

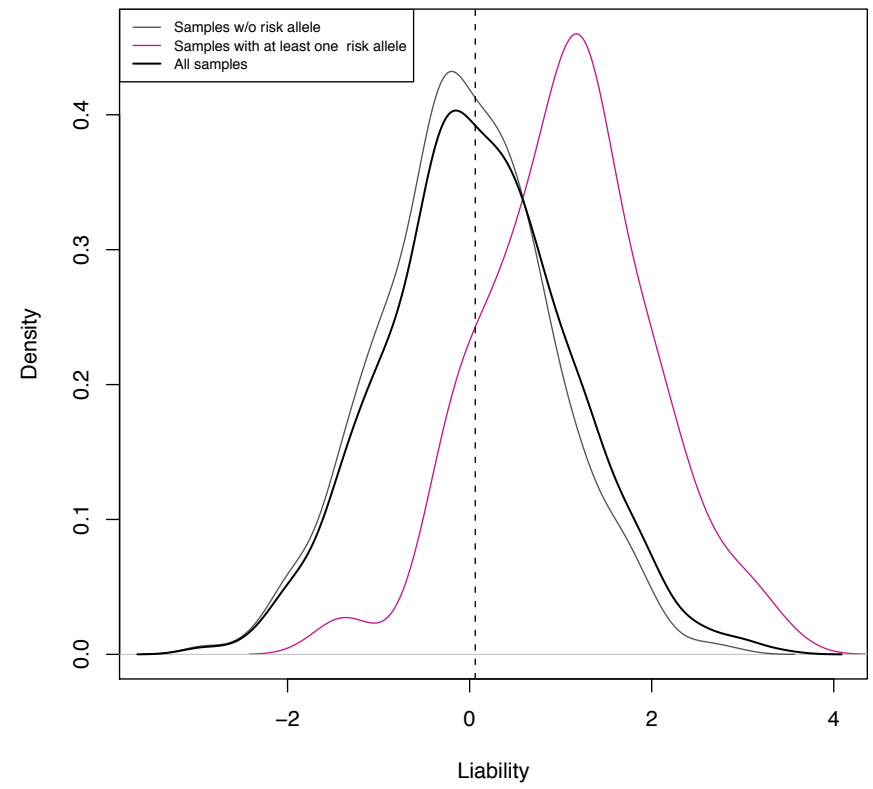

B: ITPA

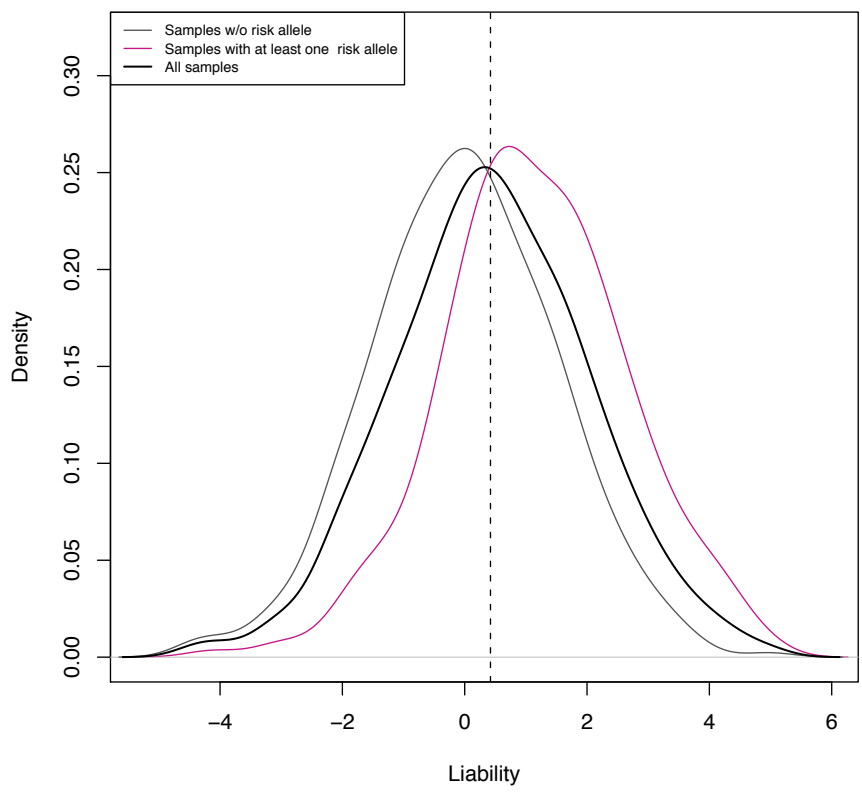

Figure S 3: Distributions of the simulated liability values for exome sequencing samples. Dashed line marks median of the distribution of all samples, which was used to classify samples to cases and controls. (A): NOD2 data, where $12 \%$ of samples were risk allele carriers. (B): ITPA data, where $39 \%$ of samples were risk allele carriers. 